\title{
Blind separation of PCMA signals based on neural network
}

\author{
WEI CHI ${ }^{1}, P E N G H U A^{1}$, and $Q I U Z E Y U^{1}$ \\ ${ }^{1}$ The Information System Engineering Institute, Information Engineering University, 450000 Zheng Zhou He Nan, China
}

\begin{abstract}
For paired carrier multiple access (PCMA) signals, a new single-channel blind separation on neural network was proposed. Firstly, the sample waveforms (three symbols) which contains different bit information are constructed, secondly, the time-frequency spectrum of each sample under the different influences of the trailing symbols is Intercepted, finally, the characteristic data of the spectrum as the input data, and the two-bit sequence in each sample as the output data to be trained, network trains these data repeatedly to complete the construction of separation model. The receiver carries on window truncation to the time-frequency spectrum of PCMA signal, neural network recognize the characteristic data of these spectrums to realizes the separation of bit sequences. Experimental results show that this algorithm has lower complexity than PSP algorithm, and the accuracy of it is close to PSP algorithm (L=5).
\end{abstract}

\section{Introduction}

In recent years, the linear mixed signal blind separation technology is used more and more widely in the field of signal processing, especially under single-channel reception, the problem of blind separation of co-frequency mixed signals has become a hot topic[1]. The single-channel blind separation technology can be applied to the problem of thirdparty reception of the PCMA (paired carrier multiple access) signal. The third party usually use singlechannel to receive PCMA signal, therefore, research on blind separation of single-channel mixed-signal is of great significance in the era.

Particle filter [2] and per-survivor processing (PSP) algorithm [3] are the main traditional algorithms to realize the separation of single channel simultaneous digital mixed signal, for the case of long constraint codes, Liao Canhui [4] and others use the idea of SOVA (soft output Viterbi algorithm) to make the PSP separation algorithm output the soft information of symbols, this method has achieved better separation performance. Aiming at the problem of high algorithm complexity, Yang Yong [5] and others use Gibbs sampling principle to reduce the complexity of the algorithm within the allowable range of guaranteed bit error rate. However, the traditional separation algorithm still has high complexity、 slow convergence speed, and unstable separation performance, this paper presents a single-channel blind separation technique of co-frequency mixed signal based on BP neural network, neural networks have multiple transport layers and each transport layer contains multiple neurons, so the trained neural network not only has stable performance but also has strong computing power[6]. it can not only make up for the lack of performance of traditional algorithms, but also the separation of information sequences is quite fast.

\section{Signal model}

For two mixed MPSK or MQAM signal (the signal's modulation type is BPSK in this paper's experiment, the same signal frequency (almost the same), the same symbol rate, the same symbol period. $T$ denotes the period of symbol[7], The response of the shaping filter and the matched filter is equivalent to the result of the channel filter $g(\cdot)$, The continuous response time of the channel is $\left[-L_{1} T, L_{1} T\right]$, after sampling at a sampling rate of $P / T$, the mixed signal can be expressed as follows:

$$
\begin{aligned}
y_{k}(l)= & \sum_{i=1}^{2} h_{i, k} e^{j\left(2 \pi l f_{i, k}(k+l / p) T+\phi_{i, k}\right)} \sum_{m=-L}^{L} s^{(i)}{ }_{k+m} g_{i}\left(-m T+l T / p-\tau_{i, k}\right) \\
& +v_{k}(l)
\end{aligned}
$$

The length channel filter memory is $L$ $\left(L=2 L_{1}+1\right), \quad v_{k}$ is a sample of white noise the mean of it is 0 , the power spectral density of it is $N_{0}$, $k$ denotes the sampling time, $s_{k+L}^{(i)}$ is the symbol entered at time $k, h_{i, k}$ denotes amplitude, $\Delta f_{i, k}$ denotes frequency offset, $\varphi_{i, k}$ denotes primary phase, $\tau_{i, k}$ denotes time delay, Since the channel parameters

\footnotetext{
* Corresponding author: author@e-mail.org
} 
of two signals change slowly with time in practical engineering applications[8], it can be assumed that the channel parameters are constant over a certain period of time, furthermore, the mixed signal in this article is further simplified as follows:

$$
\begin{aligned}
y_{k}(l)= & \sum_{i=1}^{2} h_{i} e^{j\left(2 \pi \Delta f_{i}(k+l / p) T+\varphi_{i}\right)} \sum_{m=-L}^{L} s^{(i)}{ }_{k+m} g_{i}\left(-m T+l T / p-\tau_{i}\right) \\
& +v_{k}(l)
\end{aligned}
$$

$$
\boldsymbol{y}_{G, k}=\left[y_{k}(0), y_{k}(1), \cdots, y_{k+L+G-2}(p-1)\right]^{\mathrm{T}} \quad \text { is the }
$$
received symbol sequence which is a $p(L+G-1)$ dimensional vector. After sampling the mixed signal with the sampling period of $\mathrm{Ts}=\mathrm{T} / \mathrm{p}$, the input symbol vector at $k$ moment is defined as:

$$
\begin{gathered}
\boldsymbol{s}_{i, k}(l)=e^{j 2 \pi f_{i}(\mathrm{k}+l / p) T}\left[\mathrm{~s}_{k+L_{1}}^{(\mathrm{i})}, \mathrm{s}_{k+L_{1}+1}^{(\mathrm{i})}, \cdots, \mathrm{s}_{k+L_{1}+G-1}^{(\mathrm{i})}\right]^{T} \\
\text { If } \boldsymbol{g}(l)=\left[\begin{array}{l}
\boldsymbol{g}_{1}(l) \\
\boldsymbol{g}_{2}(l)
\end{array}\right] \text {, and } \boldsymbol{s}_{k}(l)=\left[\begin{array}{l}
\boldsymbol{s}_{1, k}(l) \\
\boldsymbol{s}_{2, k}(l)
\end{array}\right], \text { the signal }
\end{gathered}
$$

model in this article is given as follow:

$$
y_{k}(l)=\boldsymbol{g}^{\boldsymbol{T}}(l) \boldsymbol{s}_{k}(l)+v_{k}(l)
$$

Therefore, we can combine the channel parameters and bit sequence to construct the Single-channel mixed signal, assuming the sample information bit sequence is $\left\{u_{r}^{(i)}\right\}$, Figure 1 shows the process of construction .

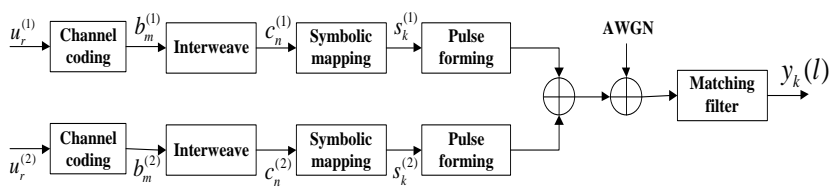

Figure1. The construction of time-domain waveform samples

Artificial neural network is a kind of mathematical model based on the simulation of nature biological neural network for information processing[9], BP neural network is a kind of training network widely used in artificial neural networks, The basic BP algorithm mainly includes forward transmission and error feedback, The entire mathematical model includes the input layer, the output layer and the hidden layer, each layer contains a number of neurons[10-11].Figure 2 shows the topology of a typical BP neural network, Figure 3 shows the model of a neuron.

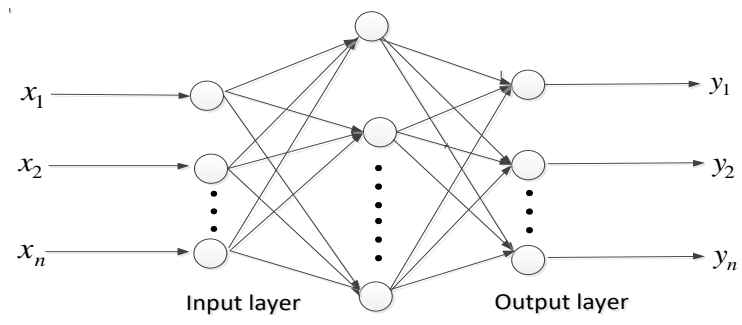

Figure2. The construction of BP neural network

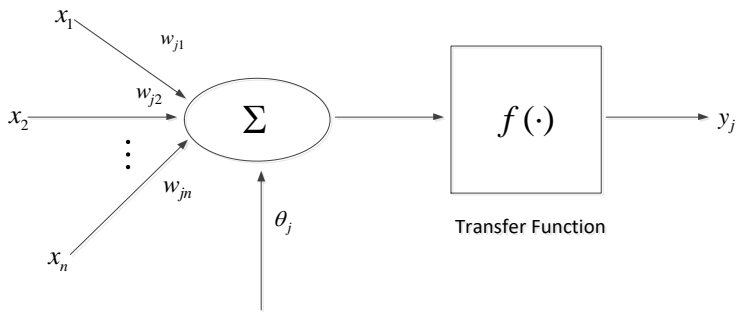

Figure3. The model of BP neuron

$X_{n}$ denotes the input sample, the output data of the output layer is $y_{p k}, k$ denotes the serial number of neurons, $E_{p}=\frac{1}{2} \sum_{k}\left(d_{p k}-y_{p k}\right)^{2}$ denotes the mean square error. the total number of samples is $N$, the adjustment formula of output layer's weight is given as follow:

$$
\begin{gathered}
\Delta_{p} w_{j k}=-\eta \frac{\partial E_{P}}{\partial w_{j k}} \\
-\eta \frac{\partial E_{P}}{\partial w_{j k}}=-\frac{\partial E_{P}}{\partial n e t_{k}} \cdot \frac{\partial n e t_{k}}{\partial w_{j k}} \\
\frac{\partial n e t_{k}}{\partial w_{j k}}=\frac{\partial}{\partial w_{j k}} \sum_{j} w_{j k} y_{p j}=y_{p j}
\end{gathered}
$$

If $-\partial E_{P} / \partial n e t_{k}=\delta_{p k}$, the adjustment formula of hidden layer's weight is given as follow:

$-\frac{\partial E_{P}}{\partial n e t_{k}}=-\frac{\partial}{\partial n e t_{k}} \frac{1}{2} \sum_{k}\left(d_{p k}-y_{p k}\right)^{2}=-\frac{\partial}{\partial n e t_{k}} \frac{1}{2} \sum_{k}\left(d_{p k}-g\left(\text { net }_{k}\right)\right)^{2}$

(8)

$$
\delta_{p k}=\left(d_{p k}-y_{p k}\right) y_{p k}\left(1-y_{p k}\right)
$$

\section{The process of extracting feature data}

Sample waveforms are constructed in the first chapter, the length of it are 3 symbols, the time-frequency spectrums of it are intercepted, then the timefrequency matrix which reflects the frequency characteristics and energy distribution of the sample signal changes with time are extracted, the timefrequency matrix are transformed into the feature matrix as the training input, When a certain sample sequence exists in any random information sequence, although the Sample waveform contains the same bit sequence, the characteristic matrix of it still will be different due to the influence of different trailing symbols, We will discuss the impact of various trailing symbol on the feature matrix comprehensively, and extract the feature matrix in different situations: 


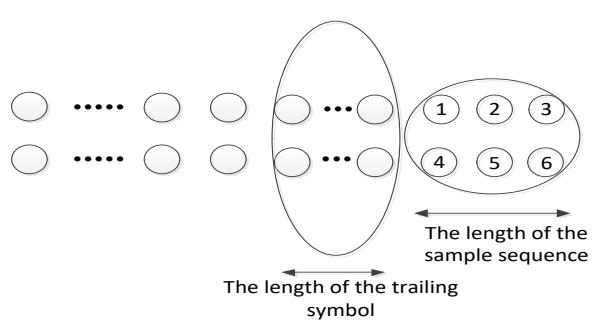

Figure4. The first type of location

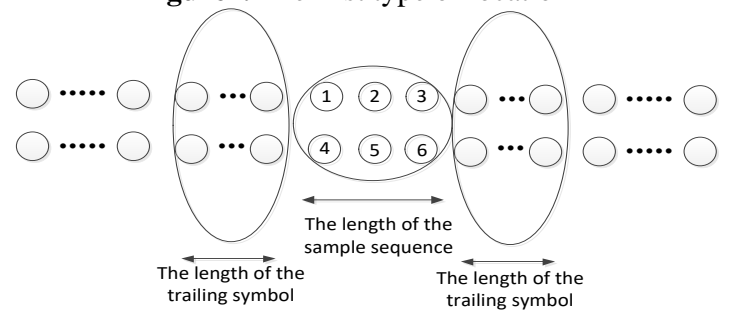

Figure5. The second type of location

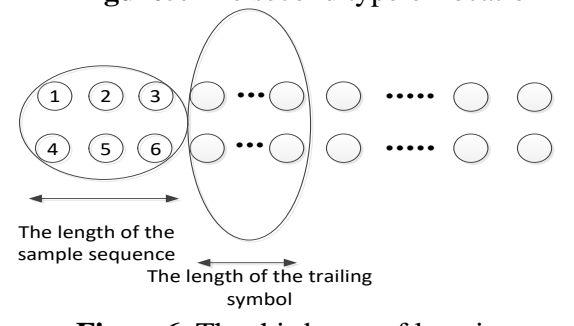

Figure6. The third type of location

According to the above pictures, we cut off the time-frequency matrix of the sample sequence respectively, Its characteristic matrix are taken as the input data in neural network, and the training output data is two-bit sequence in the sample waveform, network trains these data repeatedly to complete the construction of separation model. The receiver makes time-frequency spectrum of the received signal, and extracts the feature matrix by window length of three symbol periods, neural network realizes the separation of information sequences through the segmentation process.

\section{Simulations}

\subsection{Test 1}

We take two BPSK modulated signals as an example to simplify the experiment, the frequency offset is 0 , the oversampling factor is 2 , the delay time between two signals are 0.2 and 0.4 , the initial phases of the two channels are 1 and 2, the roll-off coefficients is 0.33 , the amplitude ratio of the two signals is 1 : 1 , The transfer functions of the hidden layer and the output layer of the network are both logarithmic S-shaped, the target error coefficient is 0.001 , the learning rate is 0.1 , the number of input nodes is 48 , the number of hidden layer nodes is 49 .Figure 7 shows the separated bit information, the data whose real part is close to 1 and imaginary part is close to 0 is bit 1 , the data whose real part is close to 0 and imaginary part is close to 1 is bit 0 , the number of experimental training is 2000 , the bit sequence length of the mixed signal is 900 .

\begin{tabular}{|c|c|c|}
\hline \multicolumn{3}{|c|}{$\boxplus 900 \times 2$ complex double } \\
\hline & 1 & 2 \\
\hline 548 & $5.7846 e-04-2 \ldots$ & $-5.0090 e-04-5 \ldots$ \\
\hline 549 & $0.9980-0.0001 \mathrm{i}$ & $0.9928-0.0001 \mathrm{i}$ \\
\hline 550 & $1.0006-0.0002 \mathrm{i}$ & $0.9947+0.0093 i$ \\
\hline 551 & $1.0002-0.0000 i$ & $-0.0077+0.00 \ldots$ \\
\hline 552 & $0.9992-0.0000 \mathrm{i}$ & $0.0002+0.0015 i$ \\
\hline 553 & $-0.0016-0.0001 i$ & $1.0063+0.0010 i$ \\
\hline 554 & $0.0020+0.0003 i$ & $1.0067-0.0027 i$ \\
\hline 555 & $0.0053-0.0001 i$ & $1.0153+0.0016 i$ \\
\hline 556 & $-0.0018-0.0000 i$ & $1.0043-0.0000 i$ \\
\hline 557 & $0.9975+0.0001 i$ & $0.0066-0.0034 i$ \\
\hline 558 & $0.0048+0.0003 i$ & $0.9901-0.0059 i$ \\
\hline 559 & $1.0019+0.0000 i$ & $1.0027+0.0028 \mathrm{i}$ \\
\hline 560 & $1.0006-0.0001 i$ & $0.9899-0.0035 i$ \\
\hline 561 & $-0.0030+0.00 \ldots$ & $0.9997-0.0026 i$ \\
\hline 562 & $0.9978-0.0001 i$ & $1.0015-0.0027 i$ \\
\hline 563 & $7.4379 e-04-1 \ldots$ & $0.0018+0.0045 i$ \\
\hline 564 & $0.9944+0.0001 i$ & $-0.0082+0.00 \ldots$ \\
\hline 565 & $0.9961+0.0001 i$ & $0.0104-0.0005 i$ \\
\hline
\end{tabular}

Figure 7. The training performance curve of BP neural network.

\subsection{Test 2}

The delay of the two signals are 0.2 and 0.4 , the initial phase and the frequency offset are both 0 , the roll-off coefficients are both 0.33 , the signal amplitude ratio is 1: 1,As shown in Figure 8, with the increase of signalto-noise ratio, the separation effect based on the neural network is close to the PSP algorithm with the symbol crosstalk length of 5 , which is better than the PSP algorithm with the symbol crosstalk length of 4 or 3. Once the neural network is trained, the network model is fixed, the separation performance is relatively stable, so its overall separation performance is superior to the traditional separation algorithm, from Tab.1, we can see that network is more efficient than PSP algorithm.

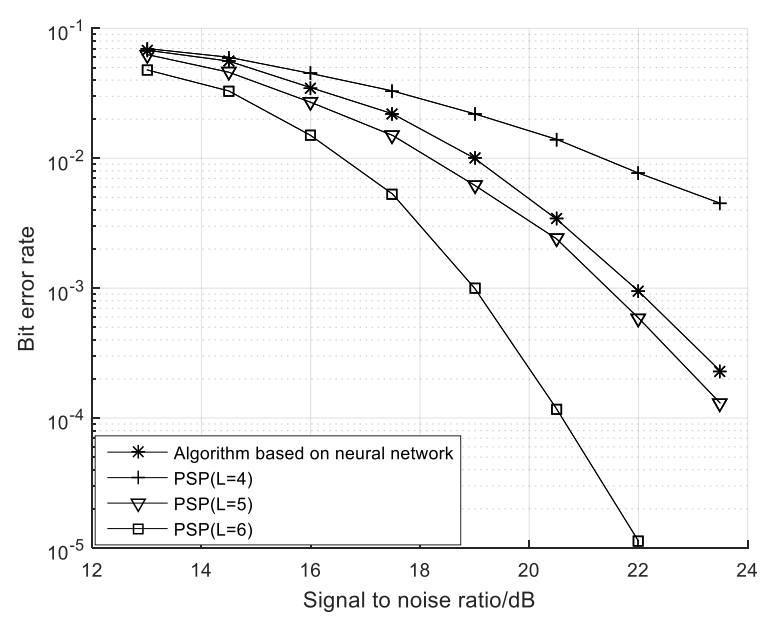

Figure8. The separation performance under different signal to noise ratio

Tab.1 The complexity of algorithm

\begin{tabular}{lllll}
\hline algorith & Conditio & additio & Multiplicat & time \\
$\mathrm{m}$ & $\mathrm{n}$ & $\mathrm{n}$ & i-on & \\
\hline
\end{tabular}




\begin{tabular}{lllll}
\hline PSP & L=4 & 11472 & 11204 & $15.16 \mathrm{~s}$ \\
& $\mathrm{~L}=5$ & 69352 & 68742 & $40.28 \mathrm{~s}$ \\
\hline Neural & $\mathrm{N}=49$ & \multirow{2}{*}{87} & \multirow{2}{*}{613} & $0.128 \mathrm{~s}$ \\
Network & $\mathrm{P}=2$ & & & \\
\hline
\end{tabular}

\subsection{Test 3}

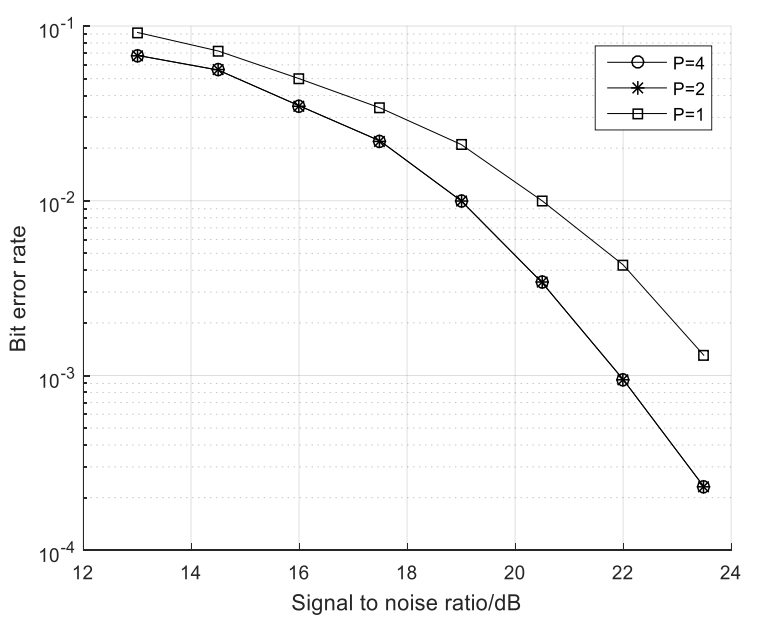

Figure9. The separation performance at different sampling rates

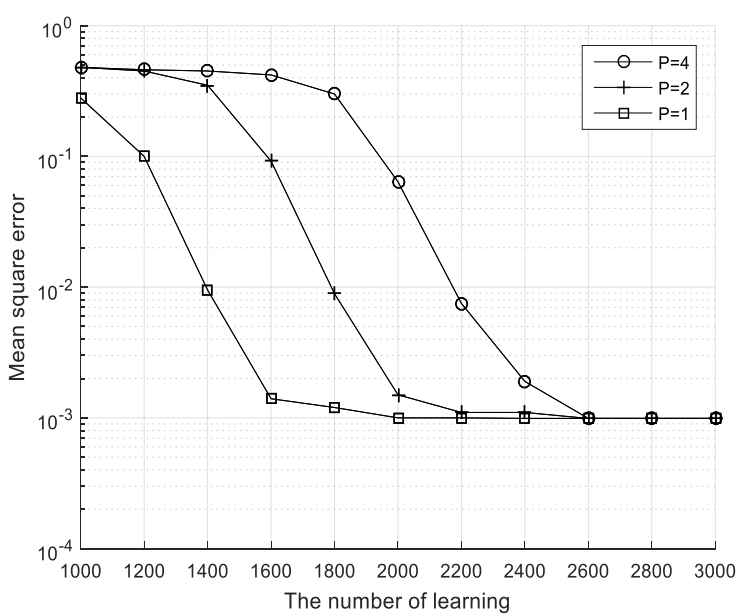

Figure10. The training performance at different sampling rates

Figure 9 shows that the greater sampling multiples, the more number of training, the increased sampling multiples increases the amount of data, the data is so difficult to be trained in network, therefore, the network will take more times to train it. Although the oversampling multiplier increases the number of training, it can get better separation performance. as shown in Figure 10, multiple sampling has better separation performance than 1-fold sampling, However, 2-fold sampling and 4-fold sampling have the same separation performance, This reason can be solved by Nyquist sampling theorem.

\section{Conclusions}

In this paper, the neural network is used to solve the problem of blind separation of PCMA signals which use BPSK modulation, the experimental results show that the accuracy of the new algorithm is close to PSP algorithm $(\mathrm{L}=5)$. This method is effective for the case where the channel parameters change slowly over a period of time, network can separate the PCMA signals whose channel parameters is same as the trained sample waveforms' parameters quickly.

\section{References}

1. YANG $\mathrm{Y}, \mathrm{ZHANGD} \mathrm{L}$, PENG $\mathrm{H}$, et a1. Frequency offset estimation in single-channel linear mixture ofhigh-order modulated signals. Acta Electronica Sinica. 43 (2015).

2. LIU K, LI H, DAI X C.Single channel blind separation of cofrequency MPSK signals[A]. Proceedings of Communications, International Information Technology[C]. 42(2006).

3. TU S L,ZHENG H,GU N.Signal-Channel blind separation of two QPSK signals using per-survivor processing[A],Processings of IEEE Asia Pacific Conference on circuits and Systems[C]. 473 (2008).

4. JIAO JP, LI YQ, WU B, et al. Research on acoustic signal recognition method for pipeline leakage with BP neural network $[\mathrm{J}]$, Chinese Journal of Scientific Instrument 37 (2016).

5. YANG Y, ZHANG D L, PENG $\mathrm{H}$. The demodulation/decoding algorithm of PCMA mixed signals based on iteration processing $[\mathrm{J}]$. Journal of Electronics \& Information Technology . 34 (2012).

6. N. Le Roux, and Y. Bengio, "Deep belief networks are compact universal approximators," Neural computation . 8 (2010).

7. LIAO C H, HUANG Y L, ZHOU S D. Joint separation and demodulation algorithm for paired carrier multiple access signals[J] . Journal on Communications . 31 (2010).

8. YANG $\mathrm{Y}$, ZHANG $\mathrm{D} \mathrm{L}$, PENG $\mathrm{H}$, et a1 . Single-channel blind separation of COfequeney modulated signals based on Gibbs sampler[J]. Journal on Communications. 36 (2015).

9. $\mathrm{Xu}$ Cao, Xiaomin Zhang, Yang Yu . Deep Learning-Based Recognition of Underwater Target, IEEE international Conference on Digital Signal Processing. 89 (2017).

10. P. Vincent, H. Larochelle, I. Lajoie et al., "Stacked denoising autoencoders: Learning useful representations in a deep net- Work with a local denoising criterion," The Journal of Machine Learning Research. 11 (2010).

11. YU YW, YIN GF, YIN Y, et al. Defect recognition for radiographic image based on deep learning network[J] . Chinese Journal of Scientific Instrument . 35(2014). 